\title{
Clinical Efficacy of Laparoscopy and Hystroscopic Dilation and Curettage Procedure in Managing Cesarean Scar Pregnancy
}

\author{
Mahin Najafian ${ }^{1}$, Raziye Mohammad Jafari ${ }^{1}$, Zorvan Jalili ${ }^{1}$, Mojgan Barati ${ }^{1}$ \& Ameneh Mozafari Garmeh ${ }^{1}$ \\ ${ }^{1}$ Faculty of Medicine, Fertility, Infertility and Perinatology Center, Ahvaz Jundishapur University of Medical \\ Sciences, Ahvaz, Iran \\ Correspondence: Ameneh Mozafari Garmeh, Faculty of Medicine, Fertility, Infertility and Perinatology Center, \\ Ahvaz Jundishapur University of Medical Sciences, Ahvaz, Iran. Tel: 98-6-1243-9152. E-mail: \\ mozafari_1366@yahoo.com
}

Received: December 5, 2019

Accepted: January 10, 2020

Online Published: March 30, 2020

doi:10.5539/jmbr.v10n1p18

URL: https://doi.org/10.5539/jmbr.v10n1p18

\begin{abstract}
Objective: To evaluate the success rate of laparoscopy and hysteroscopic Dilation \& Curettage in treating cesarean scar pregnancy.

Methods: This cross-sectional study was carried on patients suspicious to CSP referred to Imam Khomeini Hospital, Ahvaz from Jun 2016 to March 2018. Gestational site was carefully evaluated by hysteroscopy and then curettage was carried out under laparoscopic guidance. The primary outcome in the study was time interval from treatment to hCG resolution. Moreover, the secondary outcomes were vaginal bleeding time and residual mass size.

Results: In this study, 30 women with intrauterine CSP were evaluated. Of these, 5 had live embryos and 25 had dead fetuses. The success rate of treatment was $100 \%$. Only one patient needed to transfusion. The mean and median duration of Bhcg resolution were 30.9 and 28 days, respectively. Moreover, The mean and median duration of vaginal bleeding were 30.9 and 28 days, respectively. The mean level of residual mass was $9.8 \mathrm{~mm}^{3}$ (ranged from 1 to $25 \mathrm{~mm}^{3}$ ).

Conclusion: Overall, the findings of this study showed that D\&C effectively treated CSP patients (100\% success rate), reduced the time of Bhcg resolution and also reduced vaginal bleeding time.
\end{abstract}

Keywords: Dilation and Curettage, Ectopic Preganacy, Cesarean, Hysterescopy

\section{Introduction}

Pregnancy may be influenced by many factors (Valadbeigi et al., 2017). Ectopic pregnancy (EP) with an incidence of $1-2 \%$ of the total pregnancy is responsible for 3 to $4 \%$ of maternal deaths. In the United States, it has been reported that EP causes 31.8 deaths per 100,000 pregnancies. It is the most common cause of maternal death in the first trimester of pregnancy. Although Ep appears in the fallopian tube in more than $98 \%$ of cases, it rarely presented in other sites (inside the abdomen, ovaries, and in the cesarean section scar). Cesarean scar pregnancy (CSP) is referred to as replacement of a pregnancy product within the myometrium at the site of the previous cesarean section. In fact, CSP is due to a uterine endometrial defect, which can be caused by other reasons such as rough curettage, myomectomy, metroplasty, Hysteroscopy or manual removal of the placenta (Lin, Yang, Chi, Lian, Wang, Huang, Lu, Liu, \& Qiao, 2017).

There are two types of CSP: 1- Implantation of the gestational sac (GS) in the scar and progressing towards the cervical uterus; 2- Progressing the GS to the myometrium and uterine serous. Thirty-two percent of CSP have a history of more than 2 times CS, while it can be seen even in those with a history of one previous CS. It has recently been estimated that the overall incidence of CSP is 1 per 531 pregnancies in women with CS and $4.2 \%$ of those with EP. CSP incidence is rising due to an increase in CS. CSP must be diagnosed quickly, because its life-threatening nature. It could be rupture the uterus, severe bleeding, circulatory dysfunction and maternal death (Litwicka \& Greco, 2011; Petersen, Hoffmann, Larsen, \& Nielsen, 2016). There are several strategies for treating CSP. In patients who are clinically stable, conservative treatments are include uterine artery embolization with methotrexate, D \& C, curettage and hysteroscopy. D\&C under trans-abdominal ultrasound guidance is the common procedure in treating CSP. Despite the D\&C advantages including simple, low-cost, and well-therapeutic effect, it carried series dangers such as uterine perforation, bleeding, and low success rates. 
Patients treated with D\&C have a longer time to resolve the ectopic mass and B-hCG, and in some cases, such as intense hemorrhage, they require laparotomy and hysterectomy (Jurkovic, Knez, Appiah, Farahani, Mavrelos, \& Ross, 2016). According to some studies, hysteroscopic removal of CSP is an effective, safe, and non-invasive process. This method provides direct observation, less surgical operation, less hospitalization after surgery, and faster resolution of B-hCG levels.

Due to the low prevalence of CSP, there is no general agreement to choose the best treatment. Although studies have shown the benefits of hysteroscopy and D\&C in the managing of CSP, most of them designed as case studies or case series with little sample size that reduce the reliability of their results (Litwicka \& Greco, 2013). In the current study we have aimed to evaluate the success rate of hysteroscopic and D\&C in treating CSP.

\section{Material and Methods}

\subsection{Study Design}

This cross-sectional study was carried on patients suspicious to CSP referred to Imam Khomeini Hospital, Ahvaz from Jun 2016 to March 2018. Inclusion criteria was confirmed diagnosis of CSP. Patients with acute severe blood loss, hypovolemic shock, renal failure, active pelvic infection and those with coagulation disorders were excluded from the study. Patients demographic informations were extracted from the files. This study was approved by the Ethics Committee of Ahvaz Jundishapour University of Medical Sciences. Prior to entering the study, patients were provided with necessary explanations. The signed informed consent was received from all participants.

\subsection{Measurements}

EP diagnosis was performed based on history, clinical examinations, and B-HCG level. The B-HCG serum level more than $25 \mathrm{IU}$ is considered positive. CSP diagnosis confirmation was done by abdominal or vaginal ultrasonography by an expert prenatalogist. Hemodynamic factors and B-hCG serum level were checked weekly. The patients were followed-up until B-hCG resolution. Moreover, one week after treatment, residual mass size was evaluated by transvaginal sonography (TVS) (Medison Accuvix V20, Samsung, Korea).

\subsection{Interventions}

Initially, patients were treated by medical procedures including single or multiple dose of MTX. To cervical ripping, 400 milligrams of misoprostol suppository was administrated. Gestational site was carefully evaluated by hysteroscopy and then curettage was carried out under laparoscopic guidance.

\subsection{Outcomes}

The primary outcome in the study was time interval from treatment to hCG resolution. Moreover, the secondary outcomes were vaginal bleeding time and residual mass size.

\subsection{Statistical Analysis}

All variables were subjected to descriptive analysis including mean, standard deviation and frequency. The mean time to event was calculated by Kaplan-Meier plot. Computations were performed using SPSS version 19 software (Statistical Package for Social Service Inc., Chicago, IL, USA). The significant level for analyses assigned 0.05 .

\section{Results}

Table 1. Patients characteristics

\begin{tabular}{|c|c|c|c|c|c|}
\hline Variables & Median & Mean & Std. Deviation & Minimum & Maximum \\
\hline Age & 31.00 & 30.66 & 2.581 & 25.00 & 35.00 \\
\hline $\mathrm{BhCG}$ & 2887 & 8176 & 11601 & 224.0 & 49717 \\
\hline Time to BhCG resolution & 28.00 & 30.97 & 14.05 & 7.000 & 75.00 \\
\hline Time of bleeding & 30.00 & 40.48 & 20.41 & 14.00 & 120.0 \\
\hline Gravidity & 3.0 & 3.4 & 1.09 & 2.0 & 7.0 \\
\hline Parity & 2.0 & 2.24 & 0.78 & 1.0 & 4.0 \\
\hline Abortion & 0.0 & 0.31 & 0.66 & 0.0 & 3.0 \\
\hline Cesarean section & 2.0 & 2.17 & 0.84 & 1.0 & 4.0 \\
\hline Residual Mass $\left(\mathrm{mm}^{3}\right)$ & 6.0 & 9.8 & 7.4 & 1.0 & 25.0 \\
\hline Hospitalization & 3.0 & 2.9 & 0.5 & 2.0 & 4.0 \\
\hline
\end{tabular}


In this study, 30 women with intrauterine CSP were evaluated. Of these, 5 had live embryos and 25 had dead fetuses. Of the 5 patients with the live fetus, 3 patients were injected with one dose of MTX into the GS. The mean Gravidity and Parity levels in patients were 3 and 2 (range 2 to 7 and 1 to 4 ) respectively. The average abortion rate in patients was 0.3 with a maximum of 3 (Table 1). One patient was excluded during the study.

The success rate of treatment was $100 \%$. Only one patient needed to transfusion. We have not detected any procedure related complications in the studied patients. BHcg levels strictly decreased during monitoring time in all studied patients (Figure 1).

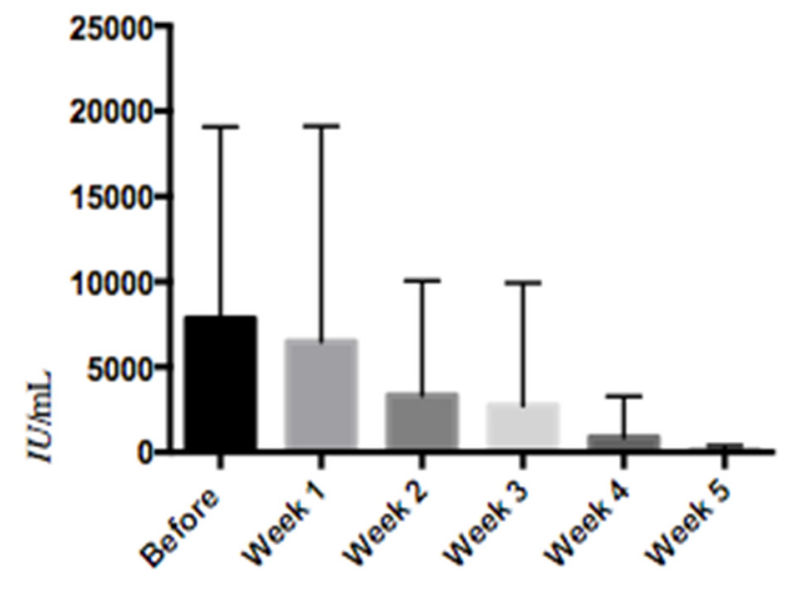

beta hCG Level

Figure 1. Changes in BHcg level

The mean and median duration of Bhcg resolution were 30.9 and 28 days, respectively. Moreover, The mean and median duration of vaginal bleeding were 30.9 and 28 days, respectively (Table 2, Figure 2). The mean level of residual mass was $9.8 \mathrm{~mm}^{3}$ (ranged from 1 to $25 \mathrm{~mm}^{3}$ ).

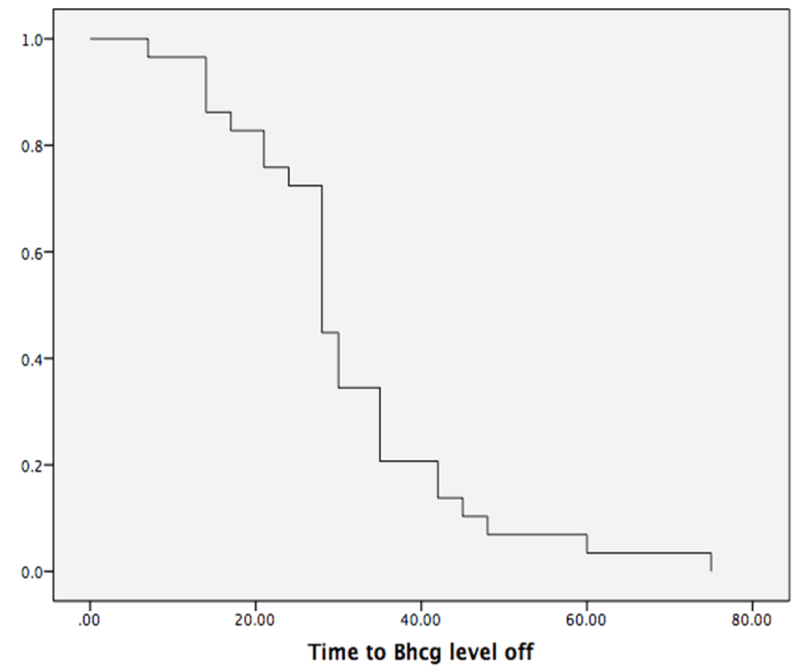

Figure 2. The Kaplan Meier chart of the Bhcg resolution time 
Table 2. Outcomes assessment

\begin{tabular}{|c|c|c|c|c|c|c|}
\hline \multirow[t]{3}{*}{ Variables } & \multicolumn{4}{|c|}{ Mean } & \multicolumn{2}{|c|}{ Median } \\
\hline & \multirow[t]{2}{*}{ Estimate } & \multicolumn{2}{|c|}{$95 \%$ Confidence Interval } & \multirow[t]{2}{*}{ Estimate } & \multicolumn{2}{|c|}{$95 \%$ Confidence Interval } \\
\hline & & Lower Bound & Upper Bound & & Lower Bound & Upper Bound \\
\hline BhCG resolution time & 30.966 & 25.850 & 36.081 & 28.000 & 25.375 & 30.625 \\
\hline Vaginal bleeding time & 40.483 & 33.054 & 47.912 & 30.000 & 29.046 & 30.954 \\
\hline
\end{tabular}

Comparison of live and dead embryos in terms of the studied factors have shown that the initial level of Bhcg, BhCG resolution time and vaginal bleeding time in patients with live fetuses were significantly higher than those with dead embryos (Table 3).

Table 3. Comparison of the studied variables between dead and live embryos

\begin{tabular}{llll}
\hline Group Statistics & Live N=5 & Dead N=24 & P value \\
\hline Bhcg & $23480 \pm 18091$ & $4987 \pm 6736$ & $\mathrm{p}<0.0001$ \\
Mass & $2.5 \pm 3$ & $6.44 \pm 7.9$ & $\mathrm{p}=0.29$ \\
Bhcg resolution Time to & $47.6 \pm 20.3$ & $27.5 \pm 9.9$ & $\mathrm{p}=0.002$ \\
Bleeding Time & $63 \pm 34.2$ & $35.79 \pm 13.06$ & $\mathrm{p}=0.004$ \\
Hospitalization & $3 \pm 0$ & $2.8 \pm 0.54$ & $\mathrm{p}=0.612$ \\
Gravity & $3.8 \pm 0.44$ & $3.4 \pm 1.17$ & $\mathrm{p}=0.484$ \\
Parity & $2.4 \pm 0.54$ & $2.2 \pm 0.83$ & $\mathrm{p}=0.629$ \\
Abortion & $0.4 \pm 0.54$ & $0.2 \pm 0.69$ & $\mathrm{p}=0.745$ \\
Cs & $2.4 \pm 0.54$ & $2.12 \pm 0.89$ & $\mathrm{p}=0.719$ \\
\hline
\end{tabular}

\section{Discussion}

Larsen and Solomon reported the first case of CSP in 1978 (Larsen \& Solomon, 1978). However, there are still no specific guideline available for the treatment of these patients. Some researchers have suggested that conservative treatment is preferred in patients with cesarean section scar greater than $2 \mathrm{~mm}$, absence abdominal pain and hemodynamically stable (Ghezzi, Lagana, Franchi, Fugazzola, \& Bolis, 2002; Khunda \& Tay, 2007). However, recent studies have shown that surgical-based therapies, including local lesion resection of the uterus, and $\mathrm{D} \& \mathrm{C}$ have many beneficial effects when compared with uterine embolization. Patients undergoing surgical treatment needs significantly less blood transfusion than patients treated by uterine embolism, shorter hospitalization time, and shorter duration of time for Bhcg resolution. Wang et al. Also showed that the recovery time is much shorter when surgical treatment performed along with systemic or topical methotrexate administration (Wang et al., 2009). In addition, preservative treatments can be associated with various complications such as uterine rupture, bleeding and the need for surgery (Stevens \& Ogburn, 2011; Jurkovic, Hillaby, Woelfer, Lawrence, Salim, \& Elson, 2003). Surgical treatment not only results in the termination of pregnancy, but also preserves fertility, reduces the amount of bleeding and shortens the duration of hospitalization. Although abdominal surgery is an effective treatment and first choice for CSP patients, it causes severe complications (Litwicka \& Greco, 2011; Chang et al., 2011; Deans \& Abbott, 2010). On the other hand, hysteroscopic surgery can reduce the incidence of hemorrhage by identifying the location of the sac and the vascular distribution surrounding the gestational implant (Deans \& Abbott, 2010; Wang, Yuen, Chao, Lee, Yen, \& Soong, 2005; Chao et al., 2005). Laparoscopic hysteroscopy can reduce the need for bladder surgeries and reduce the amount of bleeding during surgery (Li, Guo, Han, Wang, Xiong, Shen, \& Zhang, 2011). This surgery requires specialized equipment and specialized surgeons with specialized skills, which makes it difficult to do in small hospitals. Abdominal surgery is preferred in patients with confirmed uterine rupture. This can actually be the best treatment for patients with CSP, which can remove the pregnancy sac and restore uterine in order to reduce the risk of relapse (Hasegawa et al., 2005; Fylstra et al., 2002). Although this procedure causes more trauma, longer recovery time and hospitalization time (Fylstra et al., 2002). Although a general consensus suggests that D\&C is not a good treatment for CSP patients, it also has a high risk of extensive bleeding and uterine rupture, but recent studies have shown that ultrasound D\&C can treat nearly $100 \%$ of patients (Liu, Sun, Cai, Xi, Yang, \& Sun, 2016).

According to the findings, the average time needed to normalization the Bhcg level and stopping vaginal bleeding was 30.9 and 40.4 days, respectively. In a recent study, Liu and colleagues evaluated the success of 
D\&C in managing patients with CSP and found that the treatment was successful in $97.6 \%$, and their findings showed that after three weeks of treatment Bhcg was unditectable. Also, 40 days after treatment, all patients had normal menstrual cycle (Liu, Sun, Cai, Xi, Yang, \& Sun, 2016). Contrary to the current study, Liu et al. Did not prescribe methotrexate in any of the patients. Qian et al. Compared hysteroscopy with curettage in treating CSP. The researchers concluded that both D\&C and hysteroscopy along with curettage are successful in treating CSP (the success rate was 95.56\%) (Qian, Huang, \& Zhu, 2015s).

\section{Conclusion}

Overall, the findings of this study showed that D\&C effectively treated CSP patients (100\% success rate), reduced the time of Bhcg resolution and also reduced vaginal bleeding time. The lack of allocation of comparative groups was the main limitations of this study, which is suggested to be addressed in future studies.

\section{Acknowledgements}

We wish to thank all our colleagues in Imam Khomeini Hospital, Ahvaz, Iran.

\section{Ethical approval}

All procedures performed in studies involving human participants were in accordance with the ethical standards of the local ethics committee of Tehran University of Medical Sciences and with the 1964 Helsinki declaration. Written informed consent was obtained from all patients and normal individuals.

\section{Conflict of interests}

The authors declare that there is no conflict of interests regarding the publication of this paper.

\section{References}

Chang et al. (2011). Resectoscopic treatment of ectopic pregnancy in previous cesarean delivery scar defect with vasopressin injection. Fertil Steril, 96, e80-2.

Chao et al. (2005). Hysteroscopic management of cesarean scar pregnancy after unsuccessful methotrexate treatment. J Minim Invasive Gynecol, 12, 374-6.

Deans, R., \& Abbott, J. (2010). Hysteroscopic management of cesarean scar ectopic pregnancy. Fertil Steril, 93, $1735-4$.

Deans, R., \& Abbott, J. (2010). Hysteroscopic management of cesarean scar ectopic pregnancy. Fertility and sterility, 93(6), 1735-40.

Fylstra et al. (2002). Ectopic preg- nancy within a cesarean delivery scar: A case report. Am J Obstet Gynecol, 187, 302-4.

Ghezzi, F., Lagana, D., Franchi, M., Fugazzola, C., \& Bolis, P. (2002). Con- servative treatment by chemotherapy and uterine arteries embolization of a cesarean scar pregnancy. Eur J Obstet Gynecol Reprod Biol, 103, 88-91.

Hasegawa et al. (2005). Limitations of conservative treatment for repeat Cesarean scar pregnancy. Ultrasound Obstet Gynecol, 25, 310-1.

Jurkovic, D., Hillaby, K., Woelfer, B., Lawrence, A., Salim, R., \& Elson, C. J. (2003). First trimester diagnosis and management of pregnancies implanted into the lower uterine segment cesarean section scar. Ultrasound Obstet Gynecol, 21, 220-7.

Jurkovic, D., Knez, J., Appiah, A., Farahani, L., Mavrelos, D., \& Ross, J. A. (2016). Surgical treatment of Cesarean scar ectopic pregnancy: Efficacy and safety of ultrasound-guided suction curettage. Ultrasound in Obstetrics \& Gynecology, 47(4), 511-7.

Khunda, A., \& Tay, J. (2007). Caesarean scar pregnancy. BJOG, 114, 1304.

Larsen, J. V., \& Solomon, M. H. (1978). Pregnancyinauterinescarsacculus: An unusual cause of postabortal hemorrhage. A case report. S Afr Med J, 53, 142-3.

Li, H., Guo, H. Y., Han, J. S., Wang, J. L., Xiong, G. W., Shen, J., \& Zhang, J. J. (2011). Endoscopic treatment of ectopic pregnancy in a cesarean scar. Journal of Minimally Invasive Gynecology, 18(1), 31-5.

Valadbeigi, T., Ahmadi Gharaei, H., ARajabi, A., Tabatabaee, H. R., Etemad, K., Soltani, M., ArabAhmadi, A., Salehnasab, C., Almasi, Z., Yaghoobi, H., Zolfizadeh, F., Ghasemi, A., Enayatrad, M., Mahdavi, S., Hajipour, M. (2017). The relationship between physical violence during pregnancy and stillbirth and neonatal mortality. Journal of Advanced Pharmacy Education \& Research, 7 (4), 450-459.

Lin, S., Yang, R., Chi, H., Lian, Y., Wang, J., Huang, S., Lu, C., Liu, P., \& Qiao, J. (2017). Increased incidence of 
ectopic pregnancy after in vitro fertilization in women with decreased ovarian reserve. Oncotarget, $8(9)$, 14570.

Litwicka, K., \& Greco, E. (2011). Caesarean scar pregnancy: A review of management options. Current Opinion in Obstetrics and Gynecology, 23(6), 415-21.

Litwicka, K., \& Greco, E. (2013). Caesarean scar pregnancy: A review of management options. Current Opinion in Obstetrics and Gynecology, 25(6), 456-61.

Liu, S., Sun, J., Cai, B., Xi, X., Yang, L., \& Sun, Y. (2016). Management of cesarean scar pregnancy using ultrasound-guided dilation and curettage. Journal of Minimally Invasive Gynecology, 23(5), 707-11.

Petersen, K. B., Hoffmann, E., Larsen, C. R., \& Nielsen, H. S. (2016). Cesarean scar pregnancy: A systematic review of treatment studies. Fertility and sterility, 105(4), 958-67.

Qian, Z. D., Huang, L. L., \& Zhu, X. M. (2015). Curettage or operative hysteroscopy in the treatment of cesarean scar pregnancy. Archives of gynecology and obstetrics, 292(5), 1055-61.

Salman, G., \& Jurkovic, D. (2017). Ectopic Pregnancy and Pregnancy of Unknown Location. InManaging Ultrasonography in Human Reproduction 2017 (pp. 215-234). Springer International Publishing.

Stevens, E. E., \& Ogburn, P. (2011). Cesarean scar ectopic pregnancy: A case report of failed combination local and systemic methotrexate management requiring surgical intervention. J Reprod Med, 56, 356-8.

Wang et al. (2009). Methotrexate therapy for cesarean section scar pregnancy with and without suction curettage. Fertil Steril, 92, 1208-13.

Wang, C. J., Yuen, L. T., Chao, A. S., Lee, C. L., Yen, C. H., \& Soong, Y. K. (2005). Caesarean scar pregnancy successfully treated by operative hysteroscopy and suction curettage. BJOG, 112, 839-40.

Wu, X., Xue, X., Wu, X., Lin, R., Yuan, Y., Wang, Q., Xu, C., He, Y., \& Hu, W. (2014). Combined laparoscopy and hysteroscopy vs. uterine curettage in the uterine artery embolization-based management of cesarean scar pregnancy: A cohort study. International Journal of Clinical and Experimental Medicine, 7(9), 2793.

\section{Copyrights}

Copyright for this article is retained by the author(s), with first publication rights granted to the journal.

This is an open-access article distributed under the terms and conditions of the Creative Commons Attribution license (http://creativecommons.org/licenses/by/4.0/). 